\title{
Inhibitory effect of salvianolic acid on inflammatory mediators of rats with collagen-induced rheumatoid arthritis
}

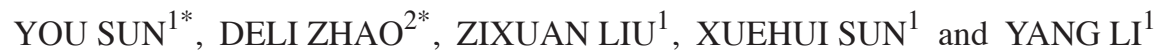 \\ Departments of ${ }^{1}$ Rheumatology and ${ }^{2}$ Radiology, The Second Affiliated Hospital of \\ Harbin Medical University, Harbin, Heilongjiang 150001, P.R. China
}

Received December 28, 2017; Accepted July 4, 2018

DOI: $10.3892 /$ etm.2018.6696

\begin{abstract}
The aim of this study was to investigate the inhibitory effect of salvianolic acid on inflammatory mediators of rats with collagen-induced rheumatoid arthritis (RA). Thirty rats were randomly divided into the normal control group, collagen-induced arthritis model group (CIA model group) and drug group (Salvianolic Acid-CIA group). In the model group, the CIA models were established through intradermal injection of collagen emulsion at the toes. At 3 weeks after the model establishment, grouped drug administration was conducted, of which salvianolic acid was given by gavage to Salvianolic Acid-CIA group. The degrees of joint swelling of each group of rats were recorded. Enzyme-linked immunosorbent assay (ELISA) was applied to detect the levels of relevant inflammatory mediators, reverse transcription-quantitative polymerase chain reaction (RT-qPCR) was used to measure the messenger RNA (mRNA) expression levels of serum necrosis factor-alpha (TNF- $\alpha$ ), interleukin-6 (IL-6) and prostaglandin E2 (PGE2), and hematoxylin and eosin staining was utilized to detect the pathological characteristics of the synovial tissues. After the establishment of models, the ankle joint swelling degree of rats in the model group was more obvious compared with that in the normal control group $(\mathrm{P}<0.01)$. After 3 weeks of drug administration, the ankle joint swelling degree of rats in the Salvianolic Acid-CIA group was alleviated compared with that in the model group $(\mathrm{P}<0.05)$. The contents of TNF- $\alpha$, IL-6 and PGE2 in Salvianolic Acid-CIA group were obviously lower than those in the model group $(\mathrm{P}<0.01)$. The mRNA expression levels of TNF- $\alpha$, IL- 6 and PGE2 in the Salvianolic Acid-CIA group were markedly lower than those in the model group. The hyperemia of rat synovial tissues in Salvianolic Acid-CIA group was obviously relieved compared with that
\end{abstract}

Correspondence to: Dr You Sun, Department of Rheumatology, The Second Affiliated Hospital of Harbin Medical University, 246 Xuefu Road, Nangang, Harbin, Heilongjiang 150001, P.R. China E-mail: youbang0901@163.com

*Contributed equally

Key words: salvianolic acid, rats, collagen-induced rheumatoid arthritis, inflammatory mediators in the CIA model group. In conclusion, the models of CIA rats are successfully established, and the results show salvianolic acid has an inhibitory effect on the RA of CIA model rats and can significantly inhibit the expression levels of relevant inflammatory mediators.

\section{Introduction}

Rheumatoid arthritis (RA) is a kind of systemic autoimmune disease, which is mainly characterized by multiple joint synovitis. Due to the combined action of environment and genetic factors, the pathogenesis of the disease is becoming more complex. Data have shown that the prevalence of RA in China is approximately $0.38 \%$, but that in European and American countries can reach nearly $2.0 \%$ (1). Since the most important complication of RA is disability, which severely influences the patient's quality of life and causes great economic pressure to society, RA has attracted the attention of scientific researchers over the last decade (2). The pathological characteristics of RA include abnormal hyperplasia of synovial tissues, aggregation of infiltrating inflammatory cells and pannus in the synovial membrane which even involves cartilage and lower-layer bone, leading to joint deformity combined with function loss.

It has been shown that the aggregation of infiltrating inflammatory cells and decompensation of synovial cell hyperplasia are closely related to the pathology of RA (3). The mechanism involved is that the aggregation of multiple inflammatory cells in the synovial tissue can induce the proliferation and thickening of synovial cells, which is involved in the injury of cartilage and lower-layer bone. Currently, the clinical medication for RA treatment mainly consists of i) non-steroidal anti-inflammatory drugs which can relieve the acute symptoms but have great side effects on the gastrointestinal tract; ii) anti-inflammatory and immunosuppressive drugs with strong efficacy, which can fundamentally treat the disease but have the shortcoming of apparent adverse reactions; thus the medication duration should not be too long and iii) biological medicine, including specific receptor antagonist and monoclonal antibody, which has obvious specificity and targeting. However, biological medicine can be degraded rapidly and have antigenicity in the body, so that the long-term outcomes are not ideal. Therefore, it is crucial to explore the complicated mechanism of RA and seek new efficient drugs (4). 
Salvianolic acid is an active ingredient of salvia miltiorrhiza Bge, which has multiple pharmacological activities, such as improving microcirculation and eliminating free radicals, exerting significant anti-atherosclerotic effects (5-7). However, there are few studies on the effects of salvianolic acid on collagen-induced RA. In this study, the rat collagen-induced arthritis (CIA) was taken as the model to investigate the impact of salvianolic acid on the arthritis of CIA rats and to preliminarily discuss its influence on relevant inflammatory mediators in rats.

\section{Materials and methods}

Materials and reagents. Salvianolic acid, bovine type II collagen, Freund's complete adjuvant (Sigma-Aldrich; Merck KGaA, Darmstadt, Germany); enzyme-linked immunosorbent assay (ELISA) kit for tumor necrosis factor- $\alpha$ (TNF- $\alpha$ ), interleukin-6 (IL-6) and prostaglandin E2 (PGE2) (Nanjing Jiancheng Bioengineering Institute, Nanjing, China); bicinchoninic acid (BCA) protein assay kit and cell lysis buffer (Beyotime Institute of Biotechnology, Nantong City, China); hematoxylin and eosin (H\&E) staining kit (Proteintech Group, Wuhan, China); synthesis of TNF- $\alpha$, IL- 6 and PGE2 primers, reverse transcription (RT) kit and quantitative polymerase chain reaction (qPCR) kit (Takara, Dalian, China) were used in the present study.

Preparation of collagen emulsion. Bovine type II collagen $(5 \mathrm{mg}$ ) was dissolved in $25 \mathrm{ml}$ acetic acid with a concentration of $0.1 \mathrm{~mol} / 1$, which was prepared into solution with a concentration of $2 \mathrm{mg} / \mathrm{ml}$, followed by preservation in a refrigerator at $4^{\circ} \mathrm{C}$ overnight. After that, $2.5 \mathrm{ml}$ Freund's complete adjuvant was added to prepare the collagen emulsion, which was stored in the refrigerator at $4^{\circ} \mathrm{C}$ until use.

Laboratory animals and grouping. Thirty female rats (4 months old, 180-200g) were randomly divided into the normal control group $(n=10)$, CIA model group $(n=10)$ and Salvianolic Acid-CIA group $(n=10)$. The models were established in the endothelium of the right posterior toes from the first day of model establishment. Rats in the normal control group were injected with $0.1 \mathrm{ml}$ normal saline, and rats in the CIA model and Salvianolic Acid-CIA groups received injection of $0.1 \mathrm{ml}$ collagen emulsion. At 7 days after the model establishment, equivalent dose of normal saline and collagen emulsion were injected via the tail vein, respectively. At 21 days after the model establishment, grouped drug administration was conducted, of which $20 \mathrm{mg} / \mathrm{kg}$ salvianolic acid was administered by gavage to rats in the Salvianolic Acid-CIA group, and $5 \mathrm{ml} / \mathrm{kg}$ normal saline was given to rats in the normal control and CIA model groups. The duration was 3 consecutive weeks. Rats were kept in cages with controlled temperature and light cycles $\left(24^{\circ} \mathrm{C}\right.$ and $12 / 12$ light cycles) and with free access to water and water. The humidity was $60 \pm 10 \%$.

The study was approved by the Ethics Committee of The Second Affiliated Hospital of Harbin Medical University (Harbin, China).

Detection of joint swelling degree of CIA rats via water capacity method. A toe volumetric measuring instrument (YLS-7A, Shan- dong Academy of Medical Science Device Station, Jinan, China) was used to detect the swelling degree
Table I. Primer sequences for RT-PCR of TNF- $\alpha$, IL-6 and PGE2 mRNAs.

\begin{tabular}{ll}
\hline Gene name & \multicolumn{1}{c}{ Primer sequences } \\
\hline TNF- $\alpha$ & F: 5'-TTTCGGGAACTAGACCTCTCACC-3' \\
& R: 5'-CTTCATGTCAGGCTTTCTGGATT-3' \\
IL-6 & F: 5'-3' CCCCCGAGACCTGAAAACCT \\
& R: 3'-5' AGTTCACCGTGGTAGTATTGTAGT \\
PGE2 & F: 5'-CCCACTCACCTGCTGCTACTC-3' \\
& R: 5'-AGAAGTGCTTGAGGTGGTTGTG-3'
\end{tabular}

F, forward; R, reverse.

of the left posterior foot, which was also known as secondary swelling; the detection was conducted before model establishment and at 21,28, 35 and 42 days after the model establishment, and the swelling degrees were calculated.

Measurement of contents of TNF- $\alpha, I L-6$ and PGE2 via ELISA. The contents of TNF- $\alpha$, IL- 6 and PGE2 in the serum of each group of rats were detected using ELISA. Experiments were performed in strict accordance with the manufacturer's protocol. During the experiments, each sample was detected 3 times, and the operation processes were repeated twice.

Detection of messenger RNA ( $m R N A$ ) expression levels of $T N F-\alpha, I L-6$ and PGE2 via RT-PCR. The synovial tissue of the rat's joint in each group was taken to extract total RNA. Viable total RNA was selected as the template for synthesis of complementary DNA (cDNA) via reverse transcription. Detailed PCR reaction conditions are: incubation at $42^{\circ} \mathrm{C}$ for $15 \mathrm{~min}$ and incubation at $95^{\circ} \mathrm{C}$ for $3 \mathrm{~min}$, followed by cooling on ice. The samples were then frozen in the refrigerator at $-80^{\circ} \mathrm{C}$ for use in subsequent experiments. Routine amplification was conducted according to the primer sequences shown in Table I.

Observation of synovial tissue pathology via $H \& E$ staining. At the end of drug administration in the Salvianolic Acid-CIA group, the rats were sacrificed after the blood was collected from the orbit. The synovial tissue at the end of tibial joint was taken out and fixed in neutral formalin with a volume fraction of $4 \%$ for $48 \mathrm{~h}$, followed by hydration, slicing and staining in accordance with the H\&E staining procedures. H\&E staining results of the rat synovial tissues in each group were observed under an ordinary light microscope (Olympus, Tokyo, Japan).

Statistical analysis. Data were presented as mean \pm standard deviation and processed using Statistical Product and Service Solutions (SPSS) 17.0 (SPSS, Inc., Chicago, IL, USA), and one-way analysis (ANOVA) of variance with SNK post hoc test was conducted for statistical analysis. $\mathrm{P}<0.05$ was considered to indicate a statistically significant difference.

\section{Results}

Measurement of swelling degree of toes. At 21 days after the model establishment, there was a significant difference in joint 
Table II. Comparison of joint swelling degrees of the left posterior foot of three groups of rats $(n=10)$.

\begin{tabular}{lcccc}
\hline & $\begin{array}{c}\text { Swelling degree of the } \\
\text { left posterior foot at } \\
21 \text { days after model } \\
\text { establishment/ml }\end{array}$ & \multicolumn{2}{c}{$\begin{array}{c}\text { Swelling degree of the rats' left posterior } \\
\text { foot after drug administration/ml }\end{array}$} \\
\cline { 3 - 5 } Groups & $0.12 \pm 0.05$ & 7 days & 14 days & 21 days \\
\hline Normal control group & $1.20 \pm 0.18^{\mathrm{a}}$ & $0.16 \pm 0.07$ & $0.19 \pm 0.09$ & $0.21 \pm 0.08$ \\
CIA model group & $1.19 \pm 0.20^{\mathrm{a}}$ & $1.28 \pm 0.16^{\mathrm{a}}$ & $1.32 \pm 0.17^{\mathrm{a}}$ & $1.33 \pm 0.19^{\mathrm{a}}$ \\
Salvianolic Acid-CIA group & $0.97 \pm 0.19^{\mathrm{a}, \mathrm{b}}$ & $0.89 \pm 0.20^{\mathrm{a}, \mathrm{b}}$ & $0.77 \pm 0.18^{\mathrm{a}, \mathrm{b}}$ \\
\hline
\end{tabular}

${ }^{\mathrm{a}} \mathrm{P}<0.01$ compared with that in the control group; ${ }^{\mathrm{b}} \mathrm{P}<0.01$ compared with that in the model group.

A

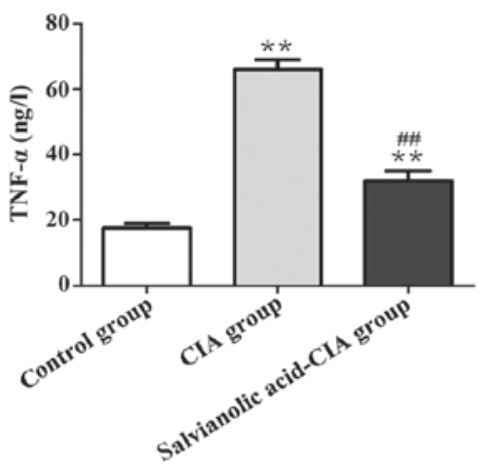

B

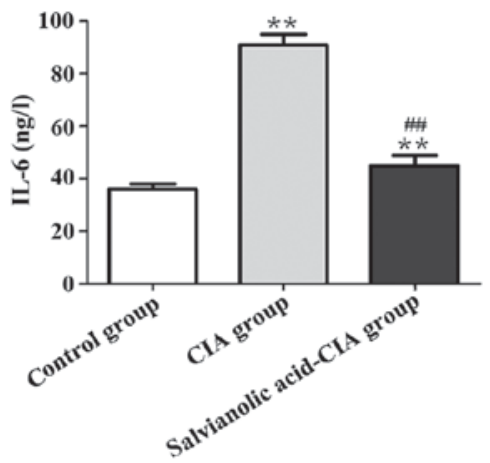

C

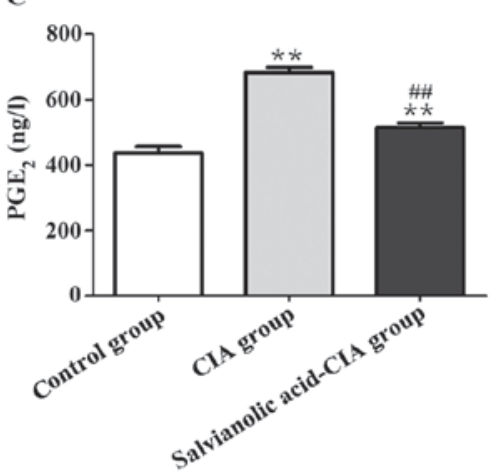

Figure 1. Effect of salvianolic acid on serum TNF- $\alpha$, IL-6 and PGE2 of CIA rats. (A) Contents of TNF- $\alpha$ in the serum of three groups. (B) Contents of IL-6 in the serum of three groups. (C) Contents of PGE2 in the serum of three groups. ${ }^{* *} \mathrm{P}<0.01$ compared with that in the control group; ${ }^{\# \#} \mathrm{P}<0.01$ compared with that in the model group.

A

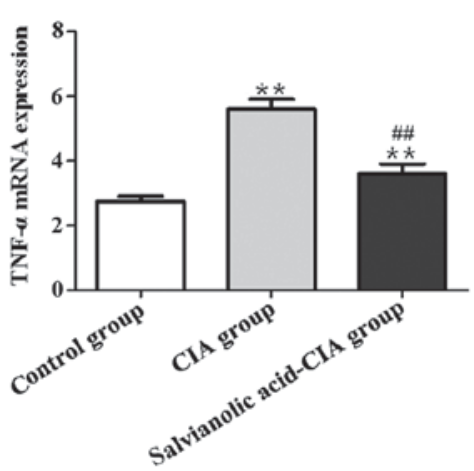

B

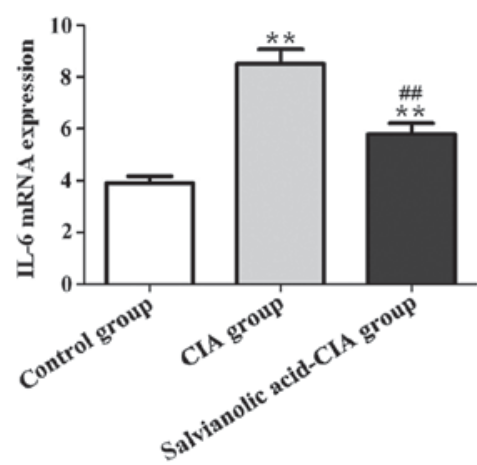

C

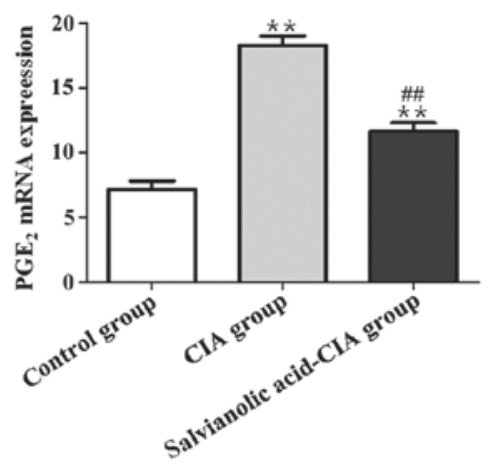

Figure 2. Effect of salvianolic acid on mRNA expression levels of TNF- $\alpha$, IL-6 and PGE2 in synovial membrane of CIA rats. (A) The mRNA expression levels of TNF- $\alpha$ in three groups. (B) The mRNA expression levels of IL- 6 in three groups. (C) The mRNA expression levels of PGE2 in three groups. ${ }^{* *} \mathrm{P}<0.01$ compared with that in the control group; ${ }^{\# \#} \mathrm{P}<0.01$ compared with that in the model group.

swelling degree in the rats' left feet between the CIA model group as well as the Salvianolic Acid-CIA and control groups $(\mathrm{P}<0.01)$, indicating that the models were established successfully. Salvianolic acid was given at 21 days after the model establishment. The left foot joint swelling degrees of the rats in the normal control, CIA model and Salvianolic Acid-CIA groups were detected at 21 days after drug administration. The results showed that there was significant difference between the Salvianolic Acid-CIA group and CIA model group $(\mathrm{P}<0.01)$ (Table II).
Effect of salvianolic acid on serum TNF- $\alpha, I L-6$ and PGE2 of CIA rats. As shown in Fig. 1, the contents of TNF- $\alpha$, IL- 6 and PGE2 in the serum in the CIA model group were significantly increased compared with those in the control group $(\mathrm{P}<0.01)$. Contents of serum TNF- $\alpha$, IL-6 and PGE2 in the Salvianolic Acid-CIA group were obviously lower than those in the model group $(\mathrm{P}<0.01)$.

Effect of salvianolic acid on mRNA expression levels of TNF- $\alpha, I L-6$ and PGE2 in the synovial membrane of CIA rats. 

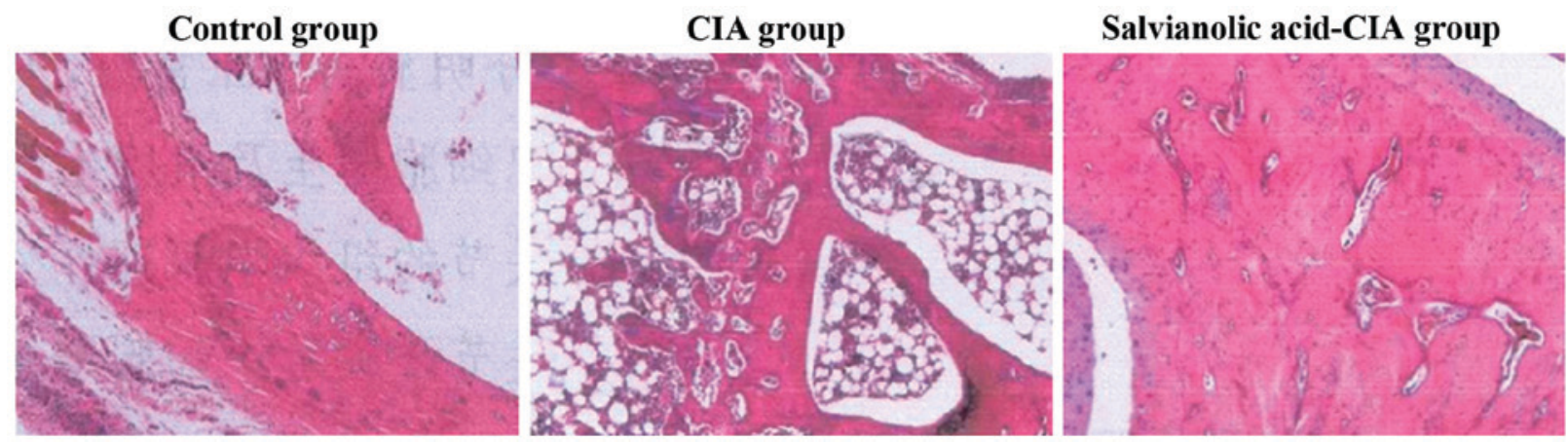

Figure 3. Effect of salvianolic acid on pathology of joint synovial membrane of CIA rats (H\&E staining, x200).

As shown in Fig. 2, the mRNA expression levels of TNF- $\alpha$, IL-6 and PGE2 in the CIA model group were notably elevated compared with those in the normal control group $(\mathrm{P}<0.01)$. The mRNA expression levels of TNF- $\alpha$, IL-6 and PGE2 in the Salvianolic Acid-CIA group were obviously decreased $(\mathrm{P}<0.01)$.

Effect of salvianolic acid on pathology of joint synovial membrane of CIA rats. As shown in Fig. 3, the H\&E staining results showed that the synovial tissues in the normal control group were arranged neatly, without hyperemia and infiltration of inflammatory cells. However, the synovial tissues in the CIA model group were arranged irregularly, with infiltrating inflammatory cells. The hyperemia and infiltration of inflammatory cells in the synovial tissues in the Salvianolic Acid-CIA group were improved significantly.

\section{Discussion}

RA is a type of autoimmune disease, whose major characteristic is chronic inflammation in the joint synovial tissue, and the main pathological manifestations are pathological hyperplasia of synovial cells associated with aggregation of infiltrating inflammatory cells, of which the cartilage and its lower-layer bone covered by the synovial membrane are involved (8); therefore, the patients' quality of life and even life are greatly threatened.

Inflammatory factors play vital roles in the occurrence and progression of RA (9). Mainly secreted by the macrophages, TNF- $\alpha$ is a crucial inflammatory factor for the inflammation of synovial tissues. It is also a master switch for the activation of inflammatory cascades in the body during the network regulation on the occurrence of RA, which, not only triggers the mononuclear macrophage to generate IL-1, IL-6 and other inflammatory factors $(10,11)$, but also causes a high expression of chemokines by the vascular endothelial cells, thus leading to the aggregation of inflammatory cells in the inflammatory sites of synovial tissues (12). The inflammatory factor IL-6 has complex functions and diversified targets, which can induce the formation of pannus and bone resorption in the progression of inflammation of the joints. In osteoporosis, IL-6 can also promote inflammation, stimulate high expression of acute phase proteins, induce anemia after the formation of hemagglutinin, cause lassitude of the hypothalamic-pituitary-adrenal (HPA) axis regulatory mechanisms and finally lead to osteoporosis.
In RA, the mechanism of action of IL-6 is that it strengthens the activities of IL-1 and TNF- $\alpha$ and induces the high expression levels of rheumatoid factor (RF) and acute phase protein in the liver. Therefore, IL-6 is crucial to the occurrence and development of RA (13). As a result, inhibiting the activity and expression of IL-6 is the key to treating RA. The activity and expression of PGE2, which is an important inflammatory mediator, play vital roles in RA. Collagenase, PGE2 and other inflammatory mediators can be synthesized and secreted by synovial fibroblasts under the stimulation of TNF- $\alpha$. However, the high expression of PGE2 can damage the cartilage and its substratum $(14,15)$. Some studies have confirmed that the occurrence and progression of RA is accompanied by the high expression of PGE2 in the serum and partial inflammatory joint cavity, causing different degrees of edema, pain and other inflammatory reactions $(8,16)$. Moreover, previous findings have indicated that whether PGE2 is highly expressed is correlated with the extent of damage to the articular cartilage and its substratum. Therefore, PGE2 plays a promoting role in stimulating damage to articular cartilage (17).

In this study, the CIA rats were taken as the model to investigate the inhibitory effect of salvianolic acid on inflammatory mediators of rats with collagen-induced RA. During the experiments, the ankle joint swelling of rats in the model group was obvious. After 3 weeks of drug administration, the ankle joint swelling degree of rats in the Salvianolic Acid-CIA group was alleviated compared with that in the model group. The ELISA results showed that the contents of serum TNF- $\alpha$, IL- 6 and PGE2 in the Salvianolic Acid-CIA group were obviously lower than those in the model group. The RT-qPCR detection results indicated that the mRNA expression levels of TNF- $\alpha$, IL-6 and PGE2 in the Salvianolic Acid-CIA group were markedly lower than those in the model group. The H\&E pathological sections showed that the hyperemia of rat synovial tissues in the Salvianolic Acid-CIA group was obviously relieved compared with that in the CIA model group, and the infiltration of inflammatory cells was decreased. In similar studies, it was found that mangiferin can ameliorate rats' inflammations of the joints in the CIA model group, and the inflammations can be alleviated by decreasing the expression levels of TNF- $\alpha$, IL-6, PGE2 and other inflammatory factors in the articular tissues (18). Kamebaaurin can lower the levels of nuclear factor kappa-lightchain-enhancer of activated B cells (NF- $\mathrm{BB}$ ), TNF- $\alpha$, PGE2 and other inflammatory factors in a dose-dependent manner to improve the status of rat arthritis (19). 
In conclusion, the models of CIA rats are successfully replicated. Moreover, salvianolic acid has an inhibitory effect on the RA of CIA model rats and can significantly inhibit the expression levels of relevant inflammatory mediators, such as TNF- $\alpha$, IL-6 and PGE2.

\section{Acknowledgements}

Not applicable.

\section{Funding}

The present study was funded by the National Natural Science Fund Project (grant no. 81202339) and Educating General Projects of Heilongjiang Province (grant no. 11511238).

\section{Availability of data and materials}

The datasets used and/or analyzed during the current study are available from the corresponding author on reasonable request.

\section{Authors' contributions}

YS prepared collagen emulsion. DZ and ZL were responsible fro animal preparation and grouping. XS and YL performed ELISA. All authors read and approved the final manuscript.

\section{Ethics approval and consent to participate}

The study was approved by the Ethics Committee of The Second Affiliated Hospital of Harbin Medical University (Harbin, China).

\section{Patient consent for publication}

Not applicable.

\section{Competing interests}

The authors declare that they have no competing interests.

\section{References}

1. Nakayama H, Yaguchi T, Yoshiya S and Nishizaki T: Resveratrol induces apoptosis MH7A human rheumatoid arthritis synovial cells in a sirtuin 1-dependent manner. Rheumatol Int 32: 151-157, 2012.

2. Cha HS, Bae EK, Ahn JK, Lee J, Ahn KS and Koh EM: Slug suppression induces apoptosis via Puma transactivation in rheumatoid arthritis fibroblast-like synoviocytes treated with hydrogen peroxide. Exp Mol Med 42: 428-436, 2010.

3. Firestein GS: Evolving concepts of rheumatoid arthritis. Nature 423: 356-361, 2003.
4. Chen YH, Du GH and Zhang JT: Salvianolic acid B protects brain against injuries caused by ischemia-reperfusion in rats. Acta Pharmacol Sin 21: 463-466, 2000.

5. Tang M, Feng W, Zhang Y, Zhong J and Zhang J: Salvianolic acid $\mathrm{B}$ improves motor function after cerebral ischemia in rats. Behav Pharmacol 17: 493-498, 2006.

6. Tang MK and Zhang JT: Salvianolic acid B inhibits fibril formation and neurotoxicity of amyloid beta-protein in vitro. Acta Pharmacol Sin 22: 380-384, 2001.

7. Cagnol S, Mansour A, Van Obberghen-Schilling E and Chambard JC: Raf-1 activation prevents caspase 9 processing downstream of apoptosome formation. J Signal Transduct 2011: 834948, 2011.

8. Funk JL, Oyarzo JN, Frye JB, Chen G, Lantz RC, Jolad SD, Sólyom AM and Timmermann BN: Turmeric extracts containing curcuminoids prevent experimental rheumatoid arthritis. J Nat Prod 69: 351-355, 2006.

9. Kiener HP, Watts GF, Cui Y, Wright J, Thornhill TS, Sköld M, Behar SM, Niederreiter B, Lu J, Cernadas M, et al: Synovial fibroblasts self-direct multicellular lining architecture and synthetic function in three-dimensional organ culture. Arthritis Rheum 62: 742-752, 2010.

10. Ferreiro E, Baldeiras I, Ferreira IL, Costa RO, Rego AC, Pereira CF and Oliveira CR: Mitochondrial- and endoplasmic reticulum-associated oxidative stress in Alzheimer's disease: From pathogenesis to biomarkers. Int J Cell Biol 2012: 735206 , 2012.

11. Car H, Zendzian-Piotrowska M, Fiedorowicz A, Prokopiuk S, Sadowska A and Kurek K: The role of ceramides in selected brain pathologies: Ischemia/hypoxia, Alzheimer disease. Postepy Hig Med Dosw (Online) 66: 295-303, 2012 (In Polish).

12. Yang Y, Karakhanova S, Soltek S, Werner J, Philippov PP and Bazhin AV: In vivo immunoregulatory properties of the novel mitochondria-targeted antioxidant SkQ1. Mol Immunol 52: 19-29, 2012.

13. Kehlen A, Thiele K, Riemann D and Langner J: Expression, modulation and signalling of IL-17 receptor in fibroblast-like synoviocytes of patients with rheumatoid arthritis. Clin Exp Immunol 127: 539-546, 2002.

14. Elkholi R, Floros KV and Chipuk JE: The role of BH3-only proteins in tumor cell development, signaling, and treatment. Genes Cancer 2: 523-537, 2011.

15. D'Orazio N, Gammone MA, Gemello E, De Girolamo M, Cusenza S and Riccioni G: Marine bioactives: Pharmacological properties and potential applications against inflammatory diseases. Mar Drugs 10: 812-833, 2012.

16. Ahmed S, Kwan K, Marotte H and Koch AE: Regulation of prostaglandin $\mathrm{E}_{2}\left(\mathrm{PGE}_{2}\right)$-induced IL-6 production by epigallocatechin-3-gallate (EGCG) in rheumatoid arthritis synovial fibroblasts. FASEB J 22: 298, 2008. http://www.fasebj.org/doi/ abs/10.1096/fasebj.22.1_supplement.298.2.

17. Akaogi J, Nozaki T, Satoh M and Yamada H: Role of PGE2 and EP receptors in the pathogenesis of rheumatoid arthritis and as a novel therapeutic strategy. Endocr Metab Immune Disord Drug Targets 6: 383-394, 2006.

18. Kumar IV, Paul BN, Asthana R, Saxena A, Mehrotra S and Rajan G: Swertia chirayita mediated modulation of interleukin1beta, interleukin-6, interleukin-10, interferon-gamma, and tumor necrosis factor-alpha in arthritic mice. Immunopharmacol Immunotoxicol 25: 573-583, 2003.

19. Lee JH, Choi JK, Noh MS, Hwang BY, Hong YS and Lee JJ: Anti-inflammatory effect of kamebakaurin in in vivo animal models. Planta Med 70: 526-530, 2004. 